\title{
Cervical Stump Fibroidpost Supra-cervical Hysterectomy
}

\author{
Sherman David Mathew, ${ }^{1}$ Betsy Abraham ${ }^{2}$ \\ ${ }^{1}$ Department of Radiology, Apollo Hospital, Muscat, India \\ ${ }^{2}$ Department of Critical Care, Royal Hospital, Muscat, India
}

\begin{abstract}
Objective: To describe of a rare case of cervical stump fibroid presenting as a late complication of supra-cervical hysterectomy.

Methods: A 68-year old-female with a history of lower abdominal pain and distention for six months with increasing intensity was referred from a local clinic to the department of radio-diagnosis for evaluation. She had a history of subtotal hysterectomy previously. On ultrasonography and computerized tomography imaging studies, a large adnexal lesion was noted suggesting the possibility of a cervical stump leiomyoma. The patient underwent an elective surgery and the diagnosis was confirmed in the context of histological findings.

Results: Patient was operated for the radiologically diagnosed large cervical stump leiomyoma which was laterhistologically confirmed with an uneventful recovery. Received: $\quad$ magnetic resonance imaging are valuable in the diagnosis of rare adnexal July 3, 2018 masses from cervical stump arising as late delayed complications of supra-

Revised:

August 8, $2018 \quad$ Keywords: Cervical stump leiomyoma, computerized tomography, magnetic

Accepted:

September 10, 2018 pISSN: 2302-1381; eISSN: 2338-4506; http://doi.org/10.15850/ijihs.v6n2.1257

IJIHS. 2018;6(2):97-9
\end{abstract}

\section{Introduction}

After supracervical hysterectomy procedure, cervical leiomyomas can occur as a rare and delayed complication These leiomymas are unusually found as mesenchymal tumours comprising $0.5-2 \%$ of all uterine leiomyomas presented dominantly with lower abdominal pain, vaginal bleeding, distension, as well as obstructive symptoms like urinary retention and constipation. ${ }^{1-3}$

Cervical myomas are generally solitary in contrast to uterine myomas and are usually encountered in the reproductive age group. ${ }^{2}$ Cervical fibroid depending on the position can clinically mimic many conditions. ${ }^{4-6}$ Excision of cervical fibroids can be risky due to close proximity to important structures in a limited

Correspondence:

Sherman David Mathew, Department of Radiology,

Apollo Hospital, Muscat 7/368C, Mavelipuram,

Kakkanad, Kerala, India

e-mail: sher_mat@yahoo.com space and has escalated the need for precise preoperative diagnosis.

In subtotal (supracervical) hysterectomy, only the upper part of uterus is removed while the cervix is left in place. On the other hand, in total hysterectomy, the entire uterus and cervix are removed. After the development of laparoscopy, supracervical hysterectomy has become a common-place, as it is shown to have significantly reduced intraoperative complications and morbidity compared to other types of hysterectomy. Despite its rare occurence, there have been cases reported cervical stump fibroids and malignancies. Other complications including, among other, prolapse, endometriosis and adhesions with the bowel have also been reported as other long term complications of cervical stump.

Current advanced imaging techniques such as ultrasonography (USG), contrast enhanced computerized tomography (CT) and magnetic resonance imaging (MRI) have valuably aided in the precise diagnosis of characterization 
of adnexal lesions especially in rare lesions arising from the cervical stump post supra cervical hysterectomy. ${ }^{7}$

\section{Case}

A 68-year-old female was referred for pelvic and abdominal evaluation by ultrasonography. Patient presented with complaints of lower abdominal pain and distention for more or less six months which was gradually increasing in severity. Patient then delivered a history of appendectomy and hysterectomy due to uterine fibroids. She was on regular medication for hypertension and diabetes mellitus.

Ultrasound image showed a well-defined pelvic mass of myometrial echogenicity with no internal calcifications or cystic spaces was found. No significant ascites was encountered while the other abdominal organs were mostly normal. From the imaging findings and clinical history, a benign entity was suggested, which was thought to be possible gastrointestinal stromal tumour. The history of hysterectomy, and initially leiomyoma was not considered in the top differential diagnosis. Further imaging was advised to further define the character and extent of the lesion and a contrast enhanced computer tomography was carried out. The patient was claustrophobic and, hence, refused magnetic resonance imaging study.

Contrast enhanced computer tomography demonstrated the presense of a large pelvic mass. The mass appeared to be in continuity with cervical stump on axial images. It is seen to demonstrate mass effect with displacement of the adjacent structures (Fig. 1).

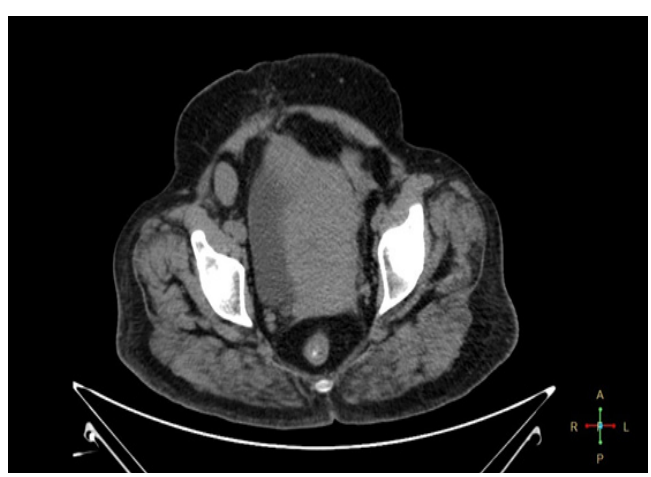

Fig. 1 CECT Abdomen, Axial View Showing Continuity of Pelvic Mass with Cervical Stump

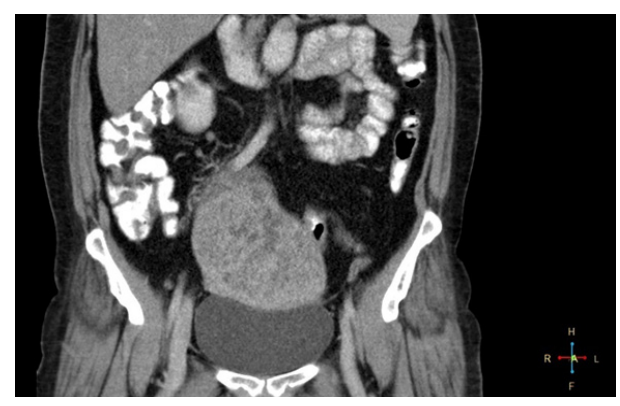

Fig. 2 CECT Abdomen, Coronal View Showing a Large Pelvic Mass of Heterogeneous Enhancement

On contrast the lesion was seen to show heterogeneous enhancement, with no specific evidence to indicate extension into urinary bladder (Fig. 2). The possibility of a cervical leiomyoma arose from the finding of cervical stump. Patient then underwent an elective excision and diagnosis was later confirmed histopathologically.

\section{Discussion}

Cervical leiomyomas are sparsely encountered mesenchymal tumours that comprise $0.5-2 \%$ of all uterine leiomyomas and may occur atypically as a delayed complication of supra cervical hysterectomy. ${ }^{1-4}$ Extensive literature search revealed no data on the incidence of such a recurrence.

The most frequently seen benign cervical mesenchymal tumours, reported with almost similar incidences, are haemangioma and leiomyoma. ${ }^{6}$ Embryonal rhabdomyosarcoma, leiomyosarcoma, undifferentiatedendocervical sarcoma, alveolar soft part sarcoma, Ewing sarcoma, malignant peripheral nerve sheath tumour and liposarcoma are most constantly reported mesenchymal malignancies, in a decreasing order. ${ }^{6}$ Leiomyomas can originate from the supra-vaginal or vaginal sections of the cervix and can be categorized as anterior, posterior, lateral and central depending on their location of genesis. ${ }^{3,4}$

Patients present predominantly with lower abdominal pain, distention, vaginal bleeding and obstructive symptoms such as urinary retention and constipation depending on the site of origin. The complications of cervical leiomyomas include pressure effects on the bladder or urethra, degenerative phenomena 
such as ulceration, inter-menstrual bleeding, pelvic cramping, prolapse with infection and torsion.

Several conditions like uterine leiomyoma, leiomyosarcoma, chronic uterine inversion, adenomyosis, tumors of ovarium, cystocoele, cervical polyps, uterine prolapse, transient myometrial contractions, lipoleiomyoma, and diffuse leiomyomatosis can clinically imitate cervical leiomyoma emphasizing the need for imaging techniques for accurate preoperative diagnosis. $^{4-8}$

Though ultrasonography is highly operator dependent, it is usually used as the preliminary investigation for evaluating the female pelvis. Transvaginal scans are more sensitive than the transabdominal views for the diagnosis of small fibroids. The transabdominal views can often be challenging in obese patients. Ultrasonography findings are that of welldefined lesions appearing hypo/isoehcoic to the normal myometrium.

The computerized tomography scan is not the investigation of choice for the evaluation of pelvic masses but can be beneficial for

\section{References}

1. Katke RJ, Thakre, Mourya, Acharya S. Cervical fibroids with its management and review of literature: an original article. Southeast Asian J Case Report Rev. 2017;6(1):5-9.

2. El-agwan AS. Lipoleiomyoma of the uterine cervix: an unusual variant of uterine leiomyoma. Egypt J Radiol Nuclear Med. 2015;46(1):211-3.

3. Taingson MC, Adze JA, Bature SB, Amina DM, Caleb M, Amina A. Vaginal myomectomy of a huge prolapsed cervical leiomyoma. Arch IntSurg. 2016;6(2):127-9.

4. Singh S, Chaudhary P . Central cervical fibroid mimicking as chronic uterine inversion: a case report. Int J Reprod Contracept Obstet Gynecol. 2013;2(4):687-8.

5. Jaouad K, Youssef B, Hanane R, Driss M, Mohamed D. Huge prolapsed cervical myoma mimicking cystocele. Saudi J Health Sci. 2015;4(2):135-7. patients with claustrophobia. Findings from computerized tomography usually present a soft tissue density lesion causing distortion of uterine contour. Some may exhibit internal calcification.

Magnetic resonance imaging is the desired imaging modality for identification of cervical fibroids and localizing their exact anatomical relations. The magnetic resonance imaging is highly sensitive in detecting various types of degeneration like hyaline, myxoid, cystic and red degeneration; calcification and malignant transformations. ${ }^{7-10}$ They appear as low to intermediate signal intensities compared to the uterine myometrium on T1 weighted and hypointense on T2 weighted imaging while on contrast imaging, variable enhancement is seen.

In conclusion, cervical leiomyoma, though rare should always be considered in cases of adnexal lesions arising in patients post supra cervical hysterectomy and advanced imaging modalities are indicated in these cases for accurate preoperative diagnosis.

6. Fadare O, Ghofrani M, Stamatakos MD, Tavassoli FA. Mesenchymal lesions of the uterine cervix. AJSP. 2006;11(3):140-52.

7. Sue W, Sarah S-B. Radiological appearances of uterine fibroids. The Indian J Radiol Imaging. 2009;19(3):222-31.

8. Teimoori B, Esmailzadeh A. A large uterine leiomyoma leading to non-puerperal uterine inversion: A case report. Int J Reprod Biomed (Yazd). 2017;15(1):55-6.

9. Khan AT, Shehmar M, Gupta JK. Uterine fibroids: current perspectives. Int J Womens Health. 2014;6(1):95-114.

10. AlharbiSR.Uterineleiomyomawithspontaneous intraleiomyoma hemorrhage, perforation, and hemoperitoneum in postmenopausal woman: computed tomography diagnosis. Avicenna J Med. 2013;3(3):81-3. 